\title{
Pan-Cancer Analysis of Atrial-Fibrillation-Related Innate Immunity Gene ANXA4
}

\author{
Tao Yan ${ }^{1 \dagger}$, Shijie Zhu ${ }^{1 \dagger}$, Yu Shi ${ }^{1,2 \dagger}$, Changming Xie ${ }^{3}$, Miao Zhu ${ }^{1}$, Yangyang Zhang ${ }^{2,4 *}$, \\ Chunsheng Wang ${ }^{1 *}$ and Changfa Guo ${ }^{1 *}$ \\ ${ }^{1}$ Department of Cardiovascular Surgery, Zhongshan Hospital, Fudan University, Shanghai, China, ${ }^{2}$ Department of \\ Cardiovascular Surgery, Shanghai East Hospital, Tongji University School of Medicine, Shanghai, China, ${ }^{3}$ Department of \\ Cardiovascular, The Eighth Affiliated Hospital, Sun Yat-sen University, Shenzhen, China, ${ }^{4}$ Department of Cardiovascular \\ Surgery, Shanghai Chest Hospital, Shanghai Jiao Tong University, Shanghai, China
}

OPEN ACCESS

Edited by:

Reto Asmis,

Wake Forest School of Medicine

United States

Reviewed by:

Tobias Schupp,

University of Heidelberg, Germany

Alessandra Cuomo,

Federico II University Hospital, Italy

*Correspondence:

Changfa Guo

guo.changfa@zs-hospital.sh.cn

Chunsheng Wang

wangchunsheng@fudan.edu.cn

Yangyang Zhang

zhangyangyang_wy@vip.sina.com

†These authors have contributed equally to this work

Specialty section:

This article was submitted to

Cardio-Oncology,

a section of the journal

Frontiers in Cardiovascular Medicine

Received: 24 May 2021

Accepted: 16 August 2021

Published: 03 September 2021

Citation:

Yan T, Zhu S, Shi Y, Xie C, Zhu M,

Zhang Y, Wang C and Guo C (2021)

Pan-Cancer Analysis of

Atrial-Fibrillation-Related Innate

Immunity Gene ANXA4.

Front. Cardiovasc. Med. 8:713983.

doi: 10.3389/fcvm.2021.713983
Background: Atrial fibrillation (AF) is the most common tachyarrhythmia around the world. Cancer is one of the main causes of death worldwide. A recent study demonstrated that cancer was associated with an increased incidence of AF. In the present study, we aimed to explore possible mechanisms and potential common therapeutic targets between AF and cancer.

Methods: Differentially expressed proteins between AF and sinus rhythm were identified utilizing proteomics analysis. Weighted gene correlation network analysis was applied to cluster proteins into different modules and investigate associations between modules and AF. Hub immune-related genes were selected via InnateDB database and verified using qRT-PCR. RNA sequencing and clinical data of 33 different cancer types were achieved from The Cancer Genome Atlas (TCGA). The correlations between ANXA4 expression and the prognosis were calculated utilizing Cox regression analysis and Kaplan-Meier survival analysis. Spearman's rank correlation test was used to assess associations between ANXA4 and immune infiltration and DNA methylation. Enrichment analysis was performed through gene ontology and gene set enrichment analysis (GSEA).

Results: ANXA4 was identified as hub immune-related gene between AF and sinus rhythm. Expression levels of ANXA4 increased in diverse cancer types. Survival analysis suggested prognostic significance of ANXA4 expression levels in various cancer types. Immune correlation analysis indicated that $A N X A 4$ expression levels were associated with tumor immune infiltration in most cancer types. ANXA4 might influence the efficacy of immunotherapy via tumor burden and microsatellite instability. GSEA results indicated that high ANXA4 expression groups were mainly enriched in peroxisome, bile acid biosynthesis, and p53 pathway.

Conclusion: ANXA4 was identified as a hub immune-related gene in AF, which has never been reported. Pan-cancer analysis indicated its potential as a novel clinical prognostic marker and therapeutic target in diverse cancer types. ANXA4 might play crucial roles in AF and cancer, and targeted therapy for ANXA4 might reduce the incidence of AF in cancer patients.

Keywords: atrial fibrillation, cancer, immune infiltration, proteomics, TCGA 


\section{INTRODUCTION}

Atrial fibrillation (AF) is the most common tachyarrhythmia worldwide, which is a significant burden for both patients and public health (1). Thromboembolism is the primary hazard of $\mathrm{AF}$, and the overall risk of stroke in patients with $\mathrm{AF}$ is five times more than that in the general population, affecting morbidity, mortality and quality of life (2). Although a variety of common risk factors have been confirmed to contribute to the development of AF, including age, sex, hypertension, obesity, and diabetes, (3) the exact pathological mechanism of AF is still unclear. Cancer, whose occurrence is a complex process with multiple risk factors, is one of the leading causes of death globally, which affects millions of people worldwide (4). A recent study based on a large population reported on the European Society of Cardiology (ESC) Congress 2020 demonstrated that cancer patients were associated with an increased incidence of AF (5). However, studies on associations between AF and cancer are still rare, which is worthy of further research to explore its possible mechanisms and potential common therapeutic targets.

Increased evidence suggests that immunity is essential both in $\mathrm{AF}$ and cancer. Expression levels of a number of immunemediated serum inflammatory biomarkers such as CRP, IL-6, and TNF- $\alpha$ have been confirmed to increase in patients with AF $(6,7)$. Histological substrates of atrial biopsies in patients with AF demonstrated that immune inflammatory cell infiltration was widespread in atrial tissues (8). A recent study indicated that M1 macrophages might be involved in the development of AF (9). Tumor microenvironment is closely related to tumor growth, metastasis, and invasion. $\mathrm{CD}^{+} \mathrm{T}$ cells, activated by interacting with antigens on antigen presenting cells, are important in the anti-tumor immune response (10). CTLA4 and PD1, expressing on $\mathrm{T}$ cells which can attenuate $\mathrm{T}$ cells response, have been applied as therapeutic targets for immunotherapy in diverse cancer types in the clinic.

In the present study, we aimed to identify immune-related proteins in $\mathrm{AF}$ using proteomics and to explore its roles in various cancer types, hoping to provide novel insights into associations between cancer and AF and reveal a potential therapeutic target to decrease the incidence of AF in cancer patients.

\section{MATERIALS AND METHODS}

\section{Data Acquisition and Processing}

Three public datasets, GSE31821, GSE41177, and GSE79768, including 11 healthy donors and $27 \mathrm{AF}$ patients, were downloaded from the Gene Expression Omnibus (GEO) database to verify the expression level of ANXA4 between sinus rhythm (SR) and AF groups. The SVA package in the R language was utilized to remove the batch effect. The gene expression of different cancer cell lines was downloaded from the Cancer Cell Line Encyclopedia (CCLE) database (11). Gene expression data of normal tissues were extracted from the Genotype-Tissue Expression (GTEx) database (12). The Cancer Genome Atlas (TCGA) was used to achieve RNA sequencing data and clinical data of 33 different cancer types. A total of 2,308 genes playing a role in the innate immune response were downloaded from the
InnateDB, a database providing a manually-curated knowledge base of the genes involved in mammalian innate immunity (13).

\section{Patients Selection}

Included in this study were ten patients with paroxysmal AF and ten with sinus rhythm (SR). All patients had complete clinical history and medical examinations, including electrocardiogram, echocardiography, coagulation function. Left atrial appendage (LAA) tissues of patients with AF and SR were collected during cardiac surgery. The use of heart tissues was in full compliance with the Declaration of Helsinki and approved by the Medical Ethics Committee of East Hospital, Tongji University. All subjects participating in this study had signed written informed consent before surgery.

\section{Protein Lysis and Quantification}

As we described before, (14) LAA tissues were cut into large pieces with scissors and washed three times with PBS. After centrifugation and discarding the supernatant, precipitation was added with an appropriate amount of SDS lysis solution and steel balls, then shook and ground in a tissue grinder until tissues were wholly broken. Protein quantification was determined with the BCA assay.

\section{Filter-Aided Sample Preparation Enzymolysis of Protein}

An appropriate amount of samples added 1M DTT solution to a final concentration of $100 \mathrm{mM}$ was incubated at $56^{\circ} \mathrm{C}$ for $1 \mathrm{~h}$. UA buffer ( $8 \mathrm{M}$ Urea, $150 \mathrm{mM}$ Tris- $\mathrm{HCl} \mathrm{pH} 8.5$ ) was taken to remove low molecular weight impurities, including SDS. IAA ( $50 \mathrm{mM}$ IAA in UA), $50 \mathrm{mM} \mathrm{NH}_{4} \mathrm{HCO}_{3}$ solution, Trypsin buffer (5 $\mu \mathrm{g}$ Trypsin in $40 \mu \mathrm{L} 50 \mathrm{mM} \mathrm{NH}_{4} \mathrm{HCO}_{3}$ solution) were also used for FASP enzymolysis of proteins. After the final filtrate was lyophilized, $50 \mu \mathrm{l}$ of $0.1 \%$ TFA was added for dissolution. The peptides were desalted by the RP-C18 solid-phase extraction column through equilibrium (rinsed once respectively by $1 \mathrm{~mL}$ of methanol, $1 \mathrm{~mL}$ of $90 \%$ acetonitrile-water solution, and $1 \mathrm{~mL}$ of double-distilled water), adsorption (fully dissolved by $1 \mathrm{~mL}$ of double distilled water and naturally adsorbed three times by gravity), washing (rinsed by $0.1 \%$ trifluoroacetic acid-water solution three times), elution (eluted by $90 \%$ acetonitrile-water solution for three times) and redissolution (redissolved by $0.1 \%$ formic acid-water solution after vacuum drying).

\section{LC-MS/MS Analysis of Enzymolysis Products}

According to the quantitative results, $1 \mu \mathrm{g}$ of the enzymatic hydrolysis products were taken for LC-MS/MS analysis. The separation was carried out by a nanoliter flow rate HPLC liquid phase system EASY-nLC1000. A 0.1\% formic acid-water solution was used for liquid phase $\mathrm{A}$, and a $0.1 \%$ formic acid-acetonitrile solution for liquid phase B. The sample was loaded by the autosampler and adsorbed on the Trap column, then separated by the Analysis column (column temperature: $50^{\circ} \mathrm{C}$, flow rate: $300 \mathrm{nl} / \mathrm{min}$ ). The relevant liquid phase gradients are as follows: 0-6 min, the linear gradient of liquid B from 1 to $5 \%$; 6-86 min, the linear gradient of liquid B from 5 to $26 \%$; $86-106 \mathrm{~min}$, the 
linear gradient of liquid B is from 26 to $40 \%$; 106-111 min, the linear gradient of liquid B is from 40 to $100 \%$; $111-116 \mathrm{~min}$, the liquid $\mathrm{B}$ is maintained at $100 \% ; 116-117 \mathrm{~min}$, the linear gradient of liquid B from 100 to $1 \%$. Separated products were analyzed by the Thermo Fisher fusion mass spectrometer. The profile mode was used for the first mass spectrometry and centroid mode for the second mass spectrometry to reduce the size of the data file.

\section{Database Search}

Thirty LC-MS/MS original files were imported into MaxQuant software (version 1.6.0.1) for database search. Label-free quantification (LFQ) analysis was conducted through the search engine Andromeda. The database was downloaded from the UniProt database. The anti-database of UniProt Homo sapiens was used to calculate the false positive rate (FDR) of peptides and proteins. MaxQuant software integrated the LFQ algorithm by extracting the isotope peaks of each peptide in each analysis. The platform calculated the protein ratio using the median of the ratio of common peptides in all analyses, which represents a relatively approximate estimate of the protein ratio. The data obtained from MaxQuant analysis was imported into Perseus (version 1.5.1.6) software for further investigation to filter out the proteins identified only by site, reverse database, and common contaminant database.

\section{Weighted Gene Correlation Network Analysis (WGCNA)}

Wgcna is a system biology method utilized to cluster genes or proteins into modules based on the interconnectivity, which can calculate correlations between modules and clinical phenotypes to identify hub genes (15). The WGCNA was utilized to construct the co-expression network based on the proteins identified using proteomics in this study. First, an appropriate soft-thresholding power $\beta$ was calculated to realize the scale-free topology with the criterion of $R^{2}>0.85$. Then the average linkage hierarchical clustering method was used to cluster proteins into different modules labeled with different colors. Each module contained at least twenty proteins, and the threshold was set as 0.25 for module merging. Pearson's correlation method was applied to determine the correlation between each module and AF. The module with a $p$-value $<0.05$ and the highest correlation coefficient was selected for further analysis.

\section{Functional Enrichment Analysis}

Gene Ontology (GO) enrichment analyses of proteins were carried out using the Database for Annotation, Visualization and Integrated Discovery (DAVID) (16) GO terms with a $p$ value $<0.05$ were considered significant enrichment. Metascape, a website developing reliable, productive and intuitive tools to analyze gene or protein lists, was also used for enrichment analysis (17). GeneMANIA, an online tool including association data of protein and genetic interactions, pathways, co-expression, co-localization, and protein domain similarity, was applied to construct the protein-protein interaction (PPI) network (18). Gene set enrichment analysis (GSEA) was performed to investigate biological signaling pathway between the high
TABLE 1 | Lists of primer sequences used for quantitative real-time PCR.

\begin{tabular}{ll}
\hline Genes & Sequences \\
\hline GAPDH & Forward: GGAGCGAGATCCCTCCAAAAT \\
& Reverse: GGCTGTTGTCATACTTCTCATGG \\
ANXA4 & Forward: GGAGGTACTGTCAAAGCTGCT \\
& Reverse: GGCAAGGACGCTAATAATGGC
\end{tabular}

ANXA4 expression group and the low ANXA4 expression group according to the median expression level of ANXA4 (19).

\section{Quantitative Real-Time PCR (qRT-PCR)}

Total cellular RNA from LAA tissues was extracted using the RNeasy ${ }^{\text {TM }}$ Mini Kit (QIAGEN, Frankfurt, Germany), following the manufacturer's instructions. The Complementary DNA (cDNA) was synthesized by reverse transcription at $42^{\circ} \mathrm{C}$ for $60 \mathrm{~min}$ and then at $95^{\circ} \mathrm{C}$ for $5 \mathrm{~min}$ with the PrimeScript ${ }^{\mathrm{TM}} \mathrm{RT}$ reagent Kit (Takara, Otsu, Japan). TB Green ${ }^{\circledR}$ Premix Ex Taq ${ }^{\text {TM }}$ II (Takara, Otsu, Japan) was applied to perform qRT-PCR at the temperature of $95^{\circ} \mathrm{C}$ for 30 seconds, followed by 40 cycles with the temperature of $95^{\circ} \mathrm{C}$ for 5 seconds and $60^{\circ} \mathrm{C}$ for 34 seconds on QuantStudio $^{\text {TM }} 5$ System (Thermo Fisher Scientific, Waltham, MA, USA). The expression of RNA levels was normalized by GAPDH, and the $2^{-\Delta \Delta C T}$ method was applied to calculate the relative expression with three independent repeats. All sequences for RNA primers (Sangon Biotech, Shanghai, China) are shown in Table 1.

\section{Survival Analysis}

Kaplan-Meier survival analysis was performed to calculate the associations between the prognosis of patients, including overall survival (OS), disease-specific survival (DSS), and progressionfree interval (PFI) and expression levels of ANXA4 in 33 different cancer types. Hazard ratio (HR) with $95 \%$ confidence intervals and $p$-value were determined using univariate Cox regression analysis and Log-rank test, respectively.

\section{Immune Infiltrating Analysis}

The Tumor Immune Evaluation Resource (TIMER) database is a comprehensive resource for systematically analyzing immune infiltrates across diverse cancer types estimated by multiple immune deconvolution methods (20). Spearman correlation analysis was used to estimate the associations between the expression levels of $A N X A 4$ and infiltrating immune scores of $\mathrm{B}$ cells, $\mathrm{CD} 4^{+} \mathrm{T}$ cells, $\mathrm{CD} 8^{+} \mathrm{T}$ cells, dendritic cells, macrophages, and neutrophils. The xCell is a webtool based on gene signatures that performs cell type enrichment analysis from gene expression data for immune cell types (21). The correlations between ANXA4 expression levels and different immune cell types were calculated utilizing xCell. Estimation of Stromal and Immune Cells in Malignant Tumor Tissues Using Expression Data (ESTIMATE) was used to predict the purity of a tumor based on infiltration of stromal cells and immunocytes, which was shown in the form of three different scores, including stromal score, immune score, and estimate score (22). The correlation between 
ANXA4 expression and these three kinds of scores was calculated using the ESTIMATE algorithm. We also examined correlations between common immune checkpoints and ANXA4 expression levels using Spearman's rank correlation test.

\section{Mutation and DNA Methylation}

Tumor mutational burden (TMB), which refers to the number of somatic mutations per megabase in the coding region, is a new genetic biomarker predicting the efficacy of immunotherapy. The correlation between the ANXA4 expression level and TMB was analyzed using Spearman's rank correlation test. Microsatellite instability (MSI) is defined as any change in the length of a microsatellite during DNA replication, resulting in the emergence of new microsatellite alleles. Spearman's rank correlation test was performed to calculate the association between ANXA4 levels and MSI. Mismatch repair (MMR) is a DNA repair mechanism, restoring the nucleotide sequence to normal in DNA molecules containing mismatched bases. We also analyzed the correlation between MMR genes ( $M L H 1$, MSH2, MSH6, PMS2, and EPCAM) and the expression level of ANXA4. DNA methylation is a form of chemical modification that can change genetic performance without changing the sequence. Under the action of methylase, DNA methylation cause changes in chromatin structure, DNA conformation, DNA stability, and the way of interaction between DNA and protein, thereby controlling gene expression. Relationships between the expression of four DNA methyltransferases (DNMT1, DNMT2, DNMT3A, and DNMT3B) and ANXA4 expression levels in different cancer types were determined.

\section{RESULTS}

\section{Baseline Characteristics of Patients}

A total of ten male paroxysmal NVAF patients were included in the study, with a mean age of $64.9 \pm 3.0$ years (range from 61 to 69 years). All patients underwent echocardiography and coronary angiography to rule out valve diseases and coronary heart disease. Detailed baseline characteristics are shown in Table 2 .

\section{Proteins Identification}

Differentially expressed proteins (DEPs) between paroxysmal NVAF and SR were identified utilizing LC-MS/MS and LFQ proteomics. A total of 1983 DEPs and 23113 unique peptides were identified for subsequent analysis.

\section{WGCNA}

Based on the proteins identified above, a total of 1983 DEPs were subjected to WGCNA. A scale-free (scale-free $R^{2}>0.85$ ) coexpression network with the soft-thresholding power $\beta=7$ was then established. The average linkage hierarchical clustering method was applied to cluster DEPs into 14 modules with different colors, including blue, dark-red, cyan, midnight-blue, dark-green, black, royal-blue, light-yellow, tan, green-yellow, red, magenta, salmon, and gray. Genes in the gray module were excluded from further analysis due to the lack of correlation (Figure 1). Correlation analysis was performed between each module and AF, showing that the red module with 419 proteins
TABLE 2 | Baseline characteristics of patients.

\begin{tabular}{|c|c|}
\hline Parameters & Values \\
\hline \multicolumn{2}{|l|}{ Gender, $n(\%)$} \\
\hline Male & $10(100 \%)$ \\
\hline Female & $0(0)$ \\
\hline Age, yr, mean $\pm S D$, (range) & $64.9 \pm 3.0$ (61 to 69$)$ \\
\hline LA diameter, mm, mean $\pm \mathrm{SD}$, (range) & $40.7 \pm 3.2$ (36 to 46$)$ \\
\hline LVEF, \%, mean \pm SD, (range) & $64.4 \pm 3.6$ (59 to 70$)$ \\
\hline $\mathrm{BMl}, \mathrm{kg} / \mathrm{m}^{2}$, mean $\pm \mathrm{SD}$, (range) & $24.6 \pm 3.5$ (18.4 to 31.2$)$ \\
\hline \multicolumn{2}{|l|}{ Medical history, $n(\%)$} \\
\hline Hypertension & $6(60 \%)$ \\
\hline Diabetic mellitus & $2(20 \%)$ \\
\hline History of preoperative stroke & $0(0)$ \\
\hline History of catheter ablation & $0(0)$ \\
\hline Valve diseases & $0(0)$ \\
\hline Coronary heart disease & $0(0)$ \\
\hline
\end{tabular}

$L A$, left atrium; $L V E F$, left ventricular ejection fraction; BMI, body mass index.

was positively correlated with AF with the highest correlation coefficient $(r=0.79, p<0.001)$.

\section{Enrichment Analysis}

DAVID online tool was utilized to perform GO functional enrichment analysis on proteins in the red module to investigate the biological effects. The significant enriched biological processes included mitochondrial electron transport from $\mathrm{NADH}$ to ubiquinone, mitochondrial respiratory chain complex I assembly, platelet degranulation, mitochondrial translational elongation, and muscle contraction (Figure 2A). For cellular components, the most significant entries were extracellular exosome, blood microparticle, mitochondrion, extracellular matrix, and mitochondrial inner membrane (Figure 2B). In addition, NADH dehydrogenase activity, actin filament binding, poly-A RNA binding, protein binding, and identical protein binding were significantly enriched in molecular function (Figure 2C).

\section{Hub Immune-Related Genes Identification and Validation}

Considering the essential roles of immune response in $\mathrm{AF}$ and cancer, we downloaded 2,308 immune-related genes playing roles in the immune response from the InnateDB. According to eigengene connectivity through WGCNA, a total of 13 hub genes were screened out with eigengene connectivity more than 0.9 in the red module. Taking the intersection between 2,308 immune-related genes and 13 hub genes, ANXA4 was identified as an immune-related gene that might regulate the pathogenesis of AF. We then performed qRT-PCR to verify the expression level of ANXA4 in AF. The results demonstrated that the ANXA4 expression level in AF was significantly higher than that in SR, which was consistent with the proteomic analysis (Figure 3). 


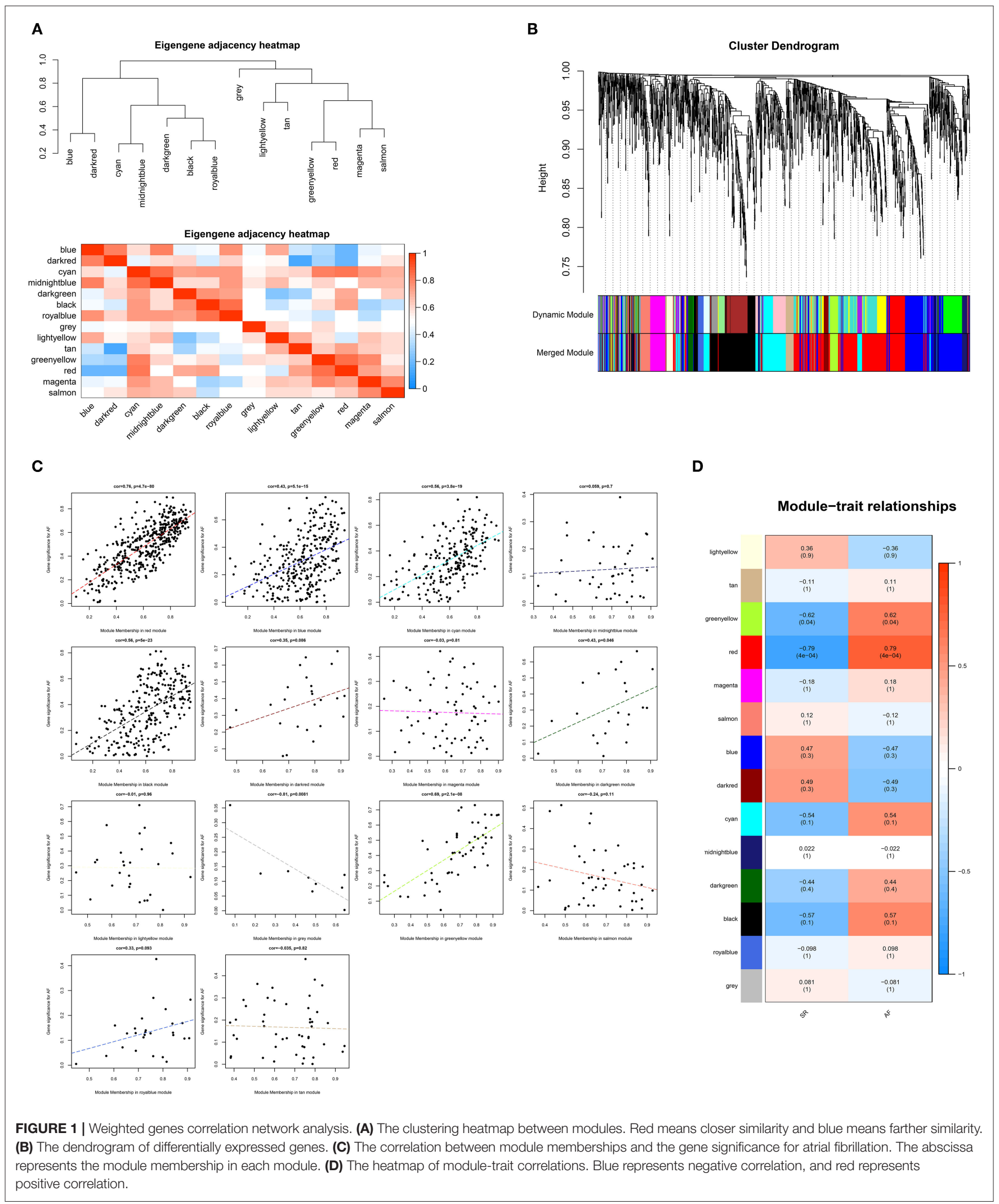




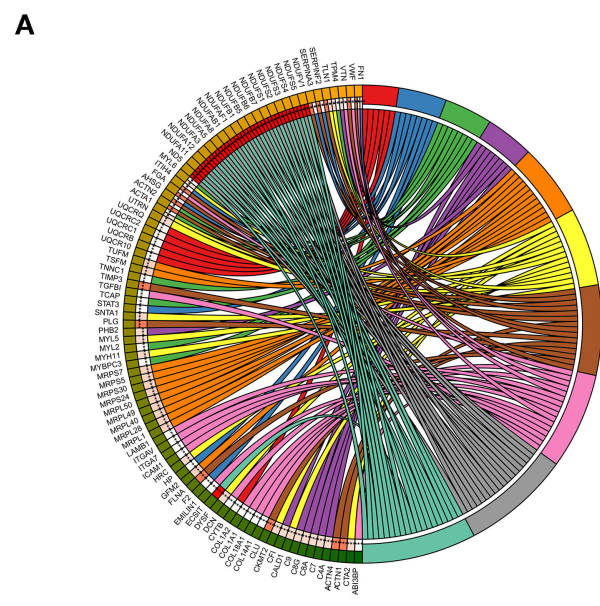

B

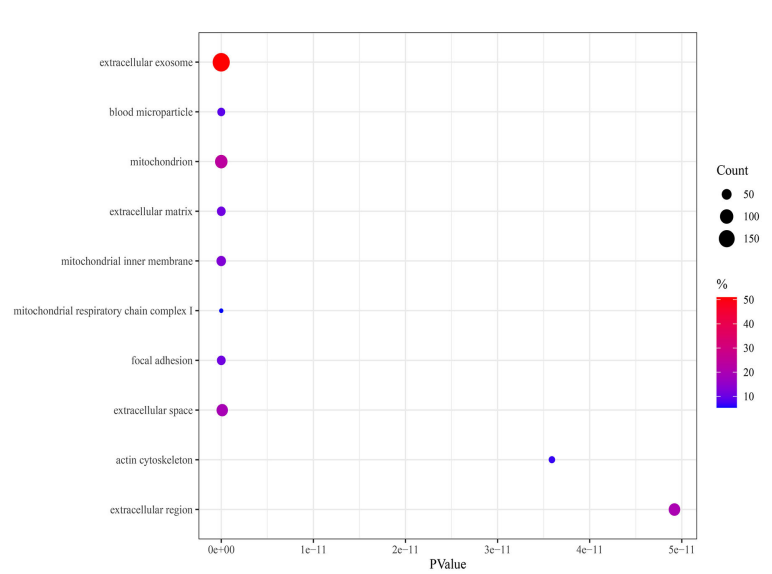

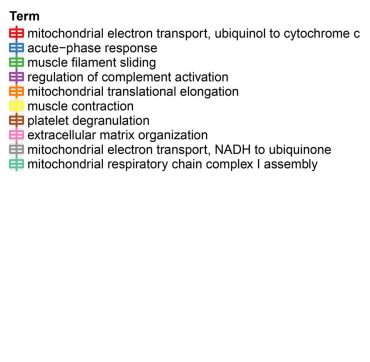

C

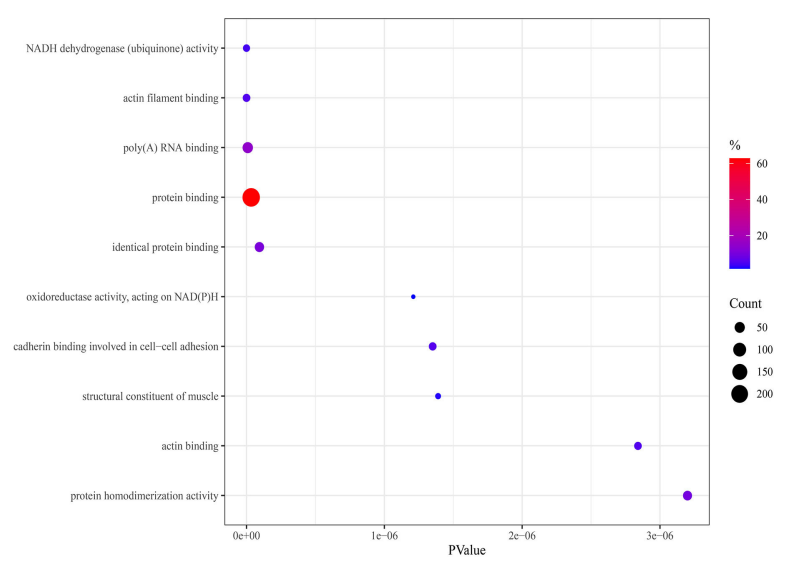

FIGURE 2 | Gene Ontology enrichment analysis. (A) Biological process. (B) Cellular component. (C) Molecular function.

\section{The Expression of ANXA4 in Different Cancer Types}

Data of diverse tumor cell lines were downloaded from the CCLE database. Expression levels of ANXA4 in different cell lines were calculated, and as shown in Figure 4A, ANXA4 expressed stably in 21 tumor cell lines. Then ANXA4 expression levels between tumor and normal tissues were analyzed using data from the TCGA database. The results demonstrated that expression levels of ANXA4 were significantly increased in most cancer types, including BLCA, CHOL, COAD, ESCA, GBM, HNSC, KIRC, KIRP, LIHC, READ, and STAD (Figure 4B). We then combined with the GTEx database to analyze the expression differences of ANXA4 between tumor tissues and normal tissues in 27 different cancer types due to small numbers of normal tissues in TCGA. The results suggested that ANXA4 expression levels were higher in CHOL, COAD, GBM, HNSC, KIRC, KIRP, LIHC, PAAD, READ, and STAD, indicating ANXA4 might act as an oncogene in many cancer types (Figure 4C).

\section{Survival Analysis}

The associations between OS of patients and ANXA4 expression levels were analyzed using Kaplan-Meier survival analysis. The results indicated that increased ANXA4 expression levels negatively correlated with OS significantly in $\operatorname{KIRP}(p<$ $0.0001)$, LAML $(p=0.0017)$, LGG $(p<0.0001)$, and UVM $(p<0.0001)$, while positively correlated with OS in KIRC $(p<0.0001)$ (Figure 5). Furthermore, high expression levels of ANXA4 were found to result in low DSS in patients with $\operatorname{KIRP}(p<0.0001)$, LGG $(p<0.0001)$, and UVM $(p<0.0001)$, while result in high DSS in patients with KIRC $(p<0.0001)$ (Supplementary Figures 1A,C). Moreover, the correlations between PFI and ANXA4 expression levels were also calculated, and we found that higher ANXA4 expression was associated with lower PFI in $\operatorname{KIRP}(p<$ $0.0001)$, LGG $(p<0.0001)$, and $\operatorname{UVM}(p=0.00042)$ (Supplementary Figures 1B,D). Results of survival analysis suggested prognostic significance of ANXA4 expression levels in diverse cancer types. 
A

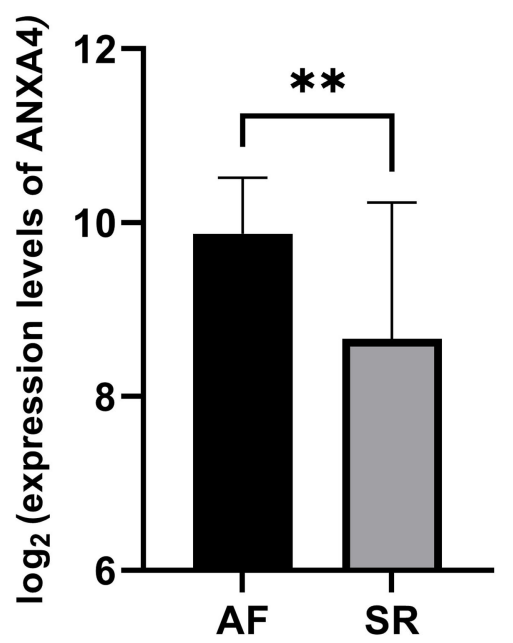

B

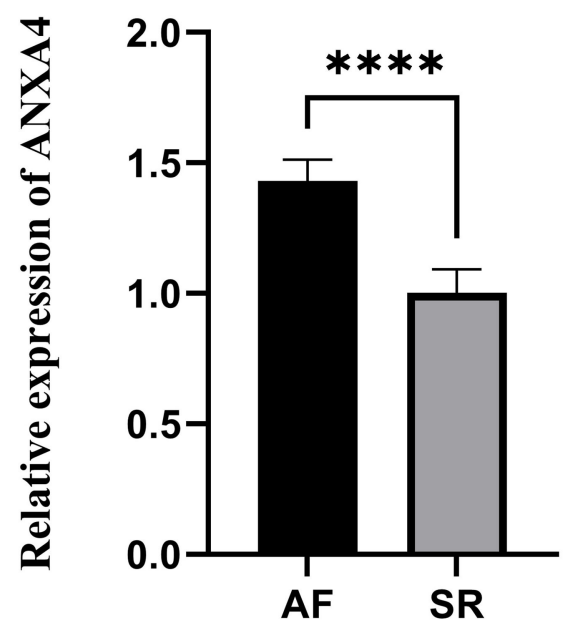

FIGURE 3 | The relative expression of ANXA4 between atrial fibrillation and sinus rhythm. (A) The relative expression of ANXA4 verified by three datasets. (B) The relative expression of ANXA4 in left atrial appendage tissues. ${ }^{\star \star} p<0.01,{ }^{\star \star \star \star} p<0.0001$.

A

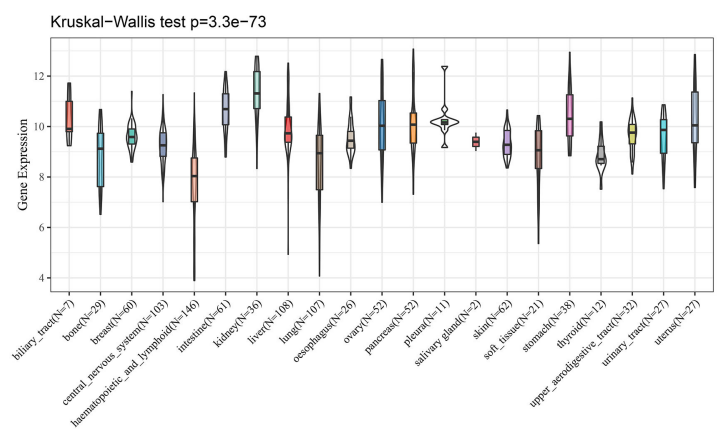

B

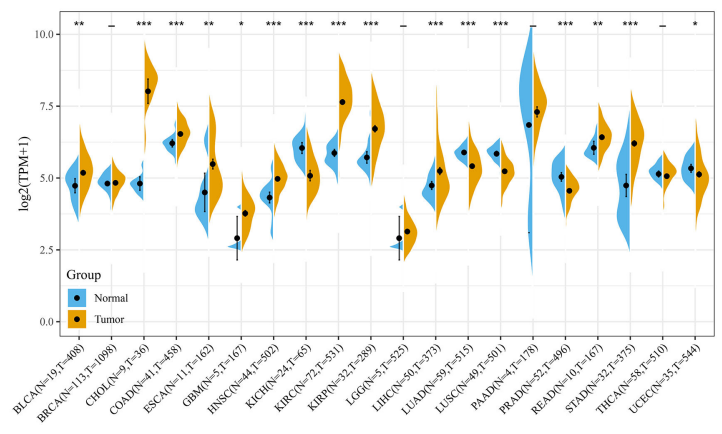

C

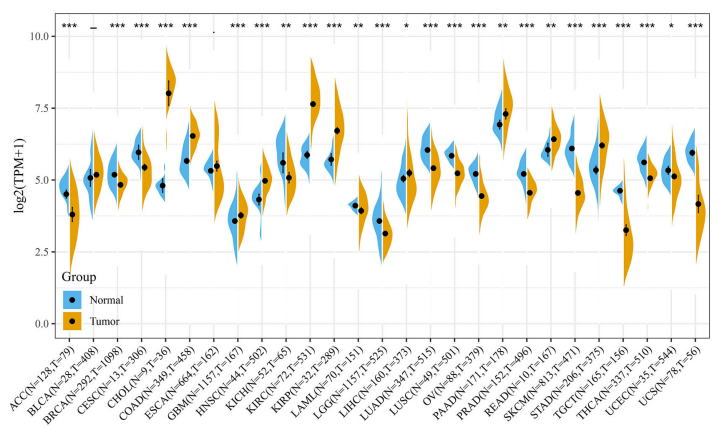

FIGURE 4 | ANXA4 expression in different cancer types. (A) Expression levels of ANXA4 in different cancer cell lines. (B) Expression levels of ANXA4 in TCGA. (C) Expression levels of ANXA4 in combination with the GTEx database and TCGA. ${ }^{*} p<0.05,{ }^{* \star} p<0.01,{ }^{* \star *} p<0.001$. 
A

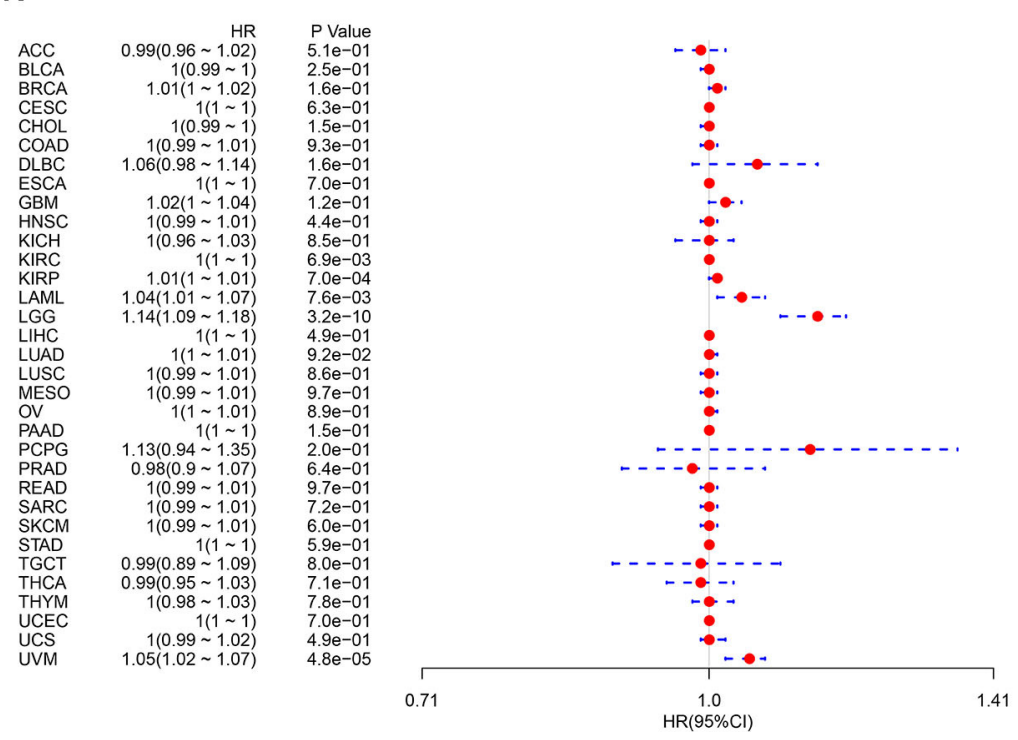

B

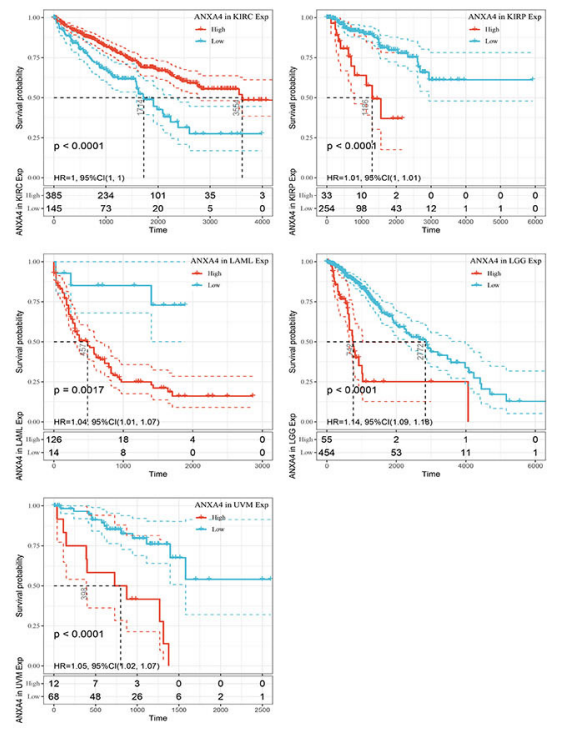

FIGURE 5 | Relationships between ANXA4 expression levels and prognosis. (A) Forest plots of overall survival (OS). (B) Kaplan-Meier curves for OS.

\section{Immune Infiltration}

To determine the role of ANXA4 in tumor infiltrating lymphocytes abundance, the TIMER database was applied to estimate correlations between ANXA4 expression and infiltration levels of different immune cells (Figures 6A,C). The results indicated that ANXA4 expression was significantly associated with $B$ cell in 15 cancer types, macrophage in 17 cancer types, dendritic cell in 20 cancer types, neutrophil in 16 cancer types, $\mathrm{CD}^{+} \mathrm{T}$ cell in 14 cancer types, and $\mathrm{CD} 8^{+} \mathrm{T}$ cell in 16 cancer types. The expression levels of ANXA4 were markedly positively correlated with infiltrating immune cells in most cancer types, especially in LGG, LIHC, PCPG, PRAD, and THCA. Only in ESCA and MESO, ANXA4 levels suggested no significant correlation with any immune cells. Further analysis utilizing the xCell online tool also demonstrated the association between ANXA4 levels and infiltrating immune cells (Figure 6B). Higher ANXA4 expression significantly decreased $\mathrm{CD} 4^{+}$Th 1 cells across 30 cancer types. ESTIMATE algorithm was used to calculate the stromal score, immune score, and estimate score of infiltration of stromal cells and immunocytes (Figures 6D-F). Cancer types significantly correlated with these three scores included BRCA, CESC, DLBC, GBM, LGG, LIHC, OV, PCPG, PRAD, TGCT, and UCS. The most three tumor types relevant to ANXA4 expression levels were DLBC, GBM, and UCS (immune score), DLBC, GBM, and UCS (estimate score), TGCT, GBM, and THYM (stromal score). Immune checkpoints were essential in immune response and immunotherapy. Correlations between expression levels of ANXA4 and common immune checkpoints were also examined (Figure 6G).

\section{TMB, MSI, MMR, and DNA Methylation}

ANXA4 was significantly correlated with TMB in BLCA, CESC, ESCA, KIRC, LIHC, LUAD, PRAD, SKCM, STAD, THCA,
THYM, UCEC, and UVM (Figure 7A). Higher expression of ANXA4 was correlated with higher TMB in BLCA, ESCA, KIRC, SKCM, STAD, THYM, and UCEC, indicating better OS and the potential of immunotherapy. The associations between ANXA4 expression levels and MSI were significant in DLBC, ESCA, HNSC, KIRC, LAML, LUAD, LUSC, PAAD, PRAD, READ, TGCT, and UCEC (Figure 7B). We then calculated the correlations between ANXA4 and MMR genes, including MLH1, MSH2, MSH6, PMS2, and EPCAM. As shown in Figure 7C, ANXA4 was associated with all five MMR genes in nine cancer types, including BLCA, BRCA, COAD, HNSC, KICH, KIRP, LIHC, READ, and THCA. These results indicated that ANXA4 might affect immunotherapy in various cancer types via TMB, MSI, or MMR. We next examined correlations between ANXA4 levels and DNA methyltransferases, including DNMT1, DNMT2, DNMT3A, and DNMT3B (Figure 7D). We found that expression levels of ANXA4 were significantly correlated with DNA methylation in diverse cancer types, especially in BRCA, HNSC, KIRP, LIHC, PCPG, and UVM.

\section{PPI Network and GSEA}

A PPI network with 21 genes centered on ANXA4 was constructed using GeneMANIA (Figure 8A). The Metascape was utilized to perform enrichment analysis on these 21 genes (Figure 8B). The significantly enriched entries included prostaglandin synthesis and regulation, negative regulation of phospholipase A2 activity, response to calcium ion, response to radiation, and regulation of protein serine/threonine kinase activity. GSEA results demonstrated that KEGG terms in the high ANXA4 expression group were mainly enriched in peroxisome, primary bile acid biosynthesis, and O-glycan biosynthesis. Hallmark enrichment terms suggested that high 


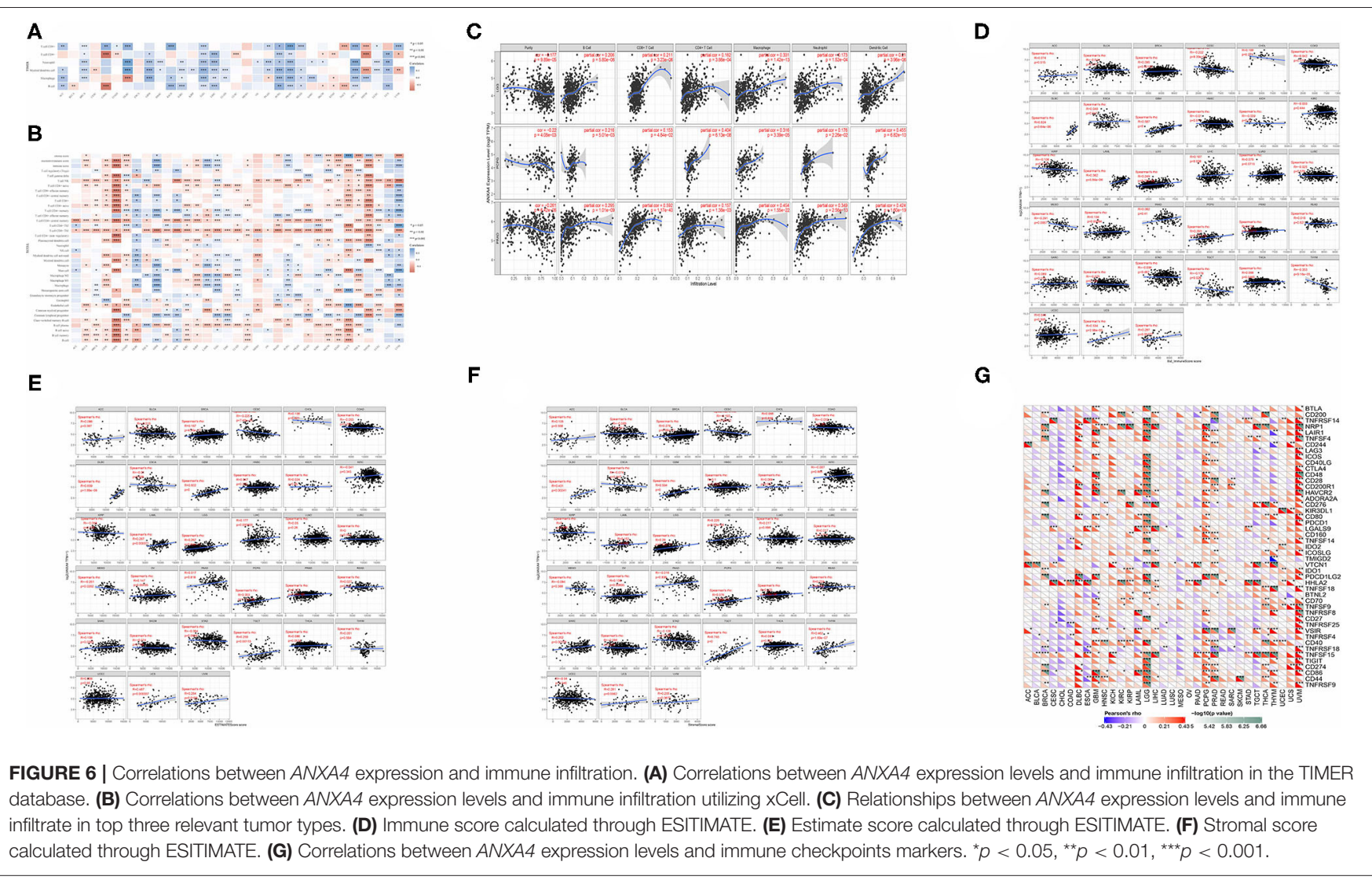

A

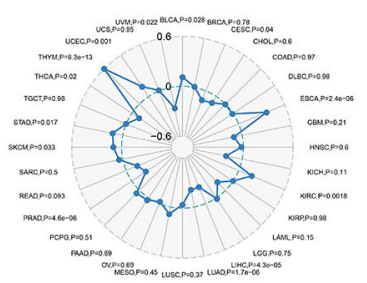

C

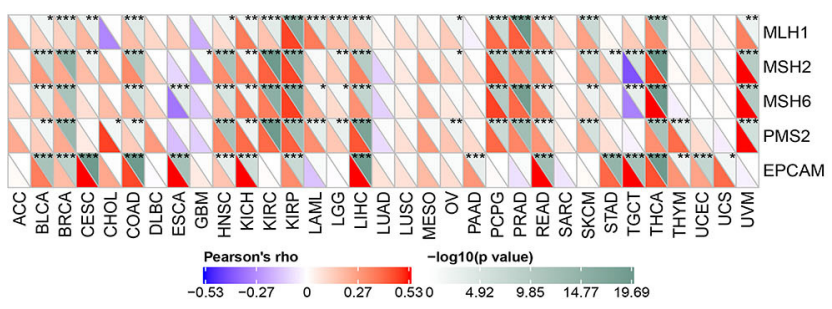

D

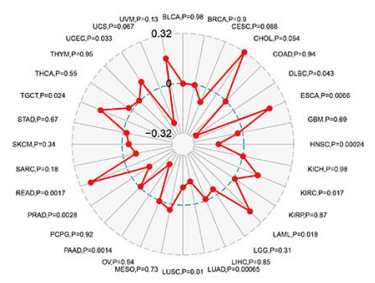

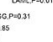

FIGURE 7 | Correlations between ANXA4 expression levels and tumor mutational burden (TMB), microsatellite instability (MSI), mismatch repair (MMR), and DNA methylation. (A) The Rader chart showing correlation between expression levels of ANXA4 and TMB. (B) The Rader chart showing correlation between expression levels of ANXA4 and MSI. (C) Correlations between ANXA4 expression levels and MMR genes (MLH1, MSH2, MSH6, PMS2, and EPCAM). ${ }^{*} P<0.05$, ${ }^{* *} p<0.01$, ${ }^{* *} p<0.001$ (D) Correlations between ANXA4 expression levels and DNA methyltransferases (red: DNMT1, blue: DNMT2, green: DNMT3A, purple: DNMT3B). 


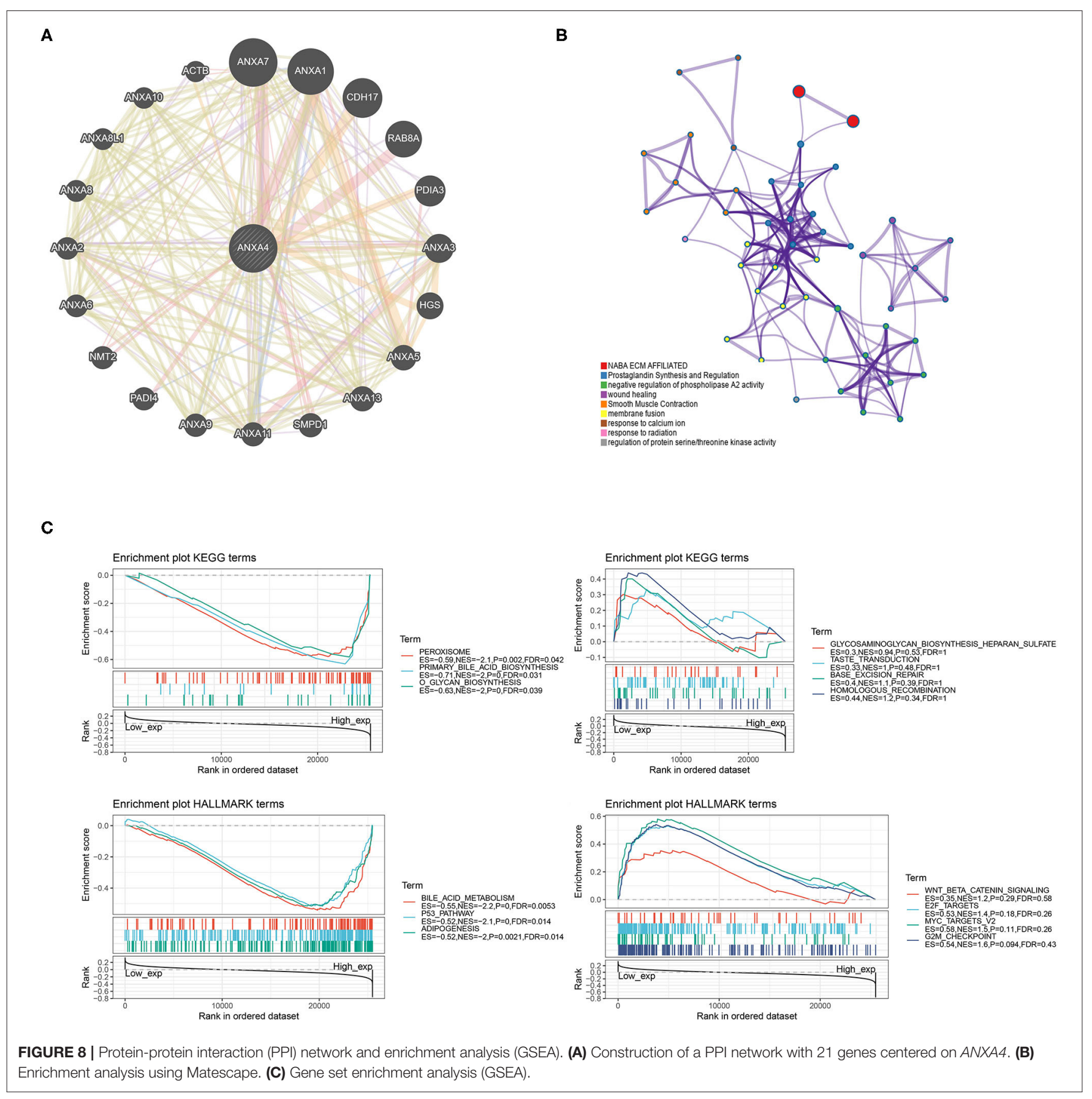

ANXA4 expression was significantly associated with bile acid metabolism, p53 pathway, and adipogenesis (Figure 8C).

\section{DISCUSSION}

AF is one of the leading causes of stroke, leading to morbidity and mortality, affecting patients' quality of life (23). Although a considerable number of studies on AF have been studied from bench to bedside, the pathophysiological mechanism of AF is still unclear. The occurrence and development of AF are related to various risk factors, such as age, sex, genetics, and obesity. Moreover, other common diseases are often associated with AF, including hypertension, diabetes, and valve diseases $(24,25)$. A recent large-population study exhibited on the ESC Congress 2020 suggested that cancer patients were associated with an increased incidence of AF. However, research on the mechanism between AF and cancer has hardly been reported.

In the present study, 20 LAA tissues underwent proteomics analysis, and a total of 1,983 DEPs were identified between AF and SR groups. WGCNA was then utilized to explore 
modules related to $\mathrm{AF}$, and the red module with the highest correlation coefficient was selected for further analysis. GO enrichment analysis demonstrated that the significantly enriched biological processes included mitochondrial electron transport from $\mathrm{NADH}$ to ubiquinone, mitochondrial respiratory chain complex I assembly, and mitochondrial translational elongation. Mitochondria, whose function is regulated by $\mathrm{Ca}^{2+}$, are the primary producers of cellular ATP in cardiac myocytes (26, 27). A recent study focused on electron microscopic features of LAA tissues demonstrated mitochondrial aggregates and an increase in mitochondria in patients with AF (28). Increased evidence suggested that mitochondrial function is impaired in patients with AF (29). Decreased expression levels of diverse enzymes contributing to mitochondrial energy metabolism were observed in AF (30). Mitochondria is also one of the main enzymatic systems producing reactive oxygen species which participate in cardiac fibrosis in $\operatorname{AF}(31,32)$. Moreover, mitochondria play central roles in cancer. Mitochondrial ubiquinol oxidation is necessary for tumor growth (33). Another study suggested that mitochondrial complex III is essential for the suppressive function of regulatory T cells (34). Mitochondria is applied as critical therapeutic targets because of its central role in cellular proliferation and death (35). Impaired mitochondrial function may be a common mechanism of AF and cancer, contributing to increased incidence of AF in cancer patients.

Considering the essential roles of immune response in $\mathrm{AF}$ and cancer, an intersection between 2308 immune-related genes downloaded from the InnateDB and 13 hub genes screened out with eigengene connectivity more than 0.9 in the red module was taken. ANXA4 was identified as the hub immune-related gene. ANXA4 is a protein-coding gene that belongs to the annexin family of calcium-dependent phospholipid-binding proteins. ANXA4 N-terminal peptide inhibits adenylyl cyclase 5 and limits beta-adrenoceptormediated prolongation of the cardiac action potential (36). However, ANXA4 has never been reported in AF, which deserves further research.

We then analyzed ANXA4 expression levels and found increased $A N X A 4$ expression in diverse cancer types, indicating that ANXA4 might act as an oncogene. Survival analysis showed prognostic significance of ANXA4. Targeted therapy for ANXA4 might relieve tumors and improved the prognosis of patients in different cancer types. Few previous studies were found between $A N X A 4$ and cancer. In ovarian carcinoma, p53 and $A N X A 4 / \mathrm{NF}-\kappa \mathrm{B}$ p50 complexes regulate cell proliferation, apoptosis, and tumor progression (37). The overexpression of Lewis(y), a component of the structure of the ANXA4 membrane protein, induces the chemoresistance of ovarian cancer cells, and ultimately promoting the progression of ovarian cancer (38). It was reported that the increased serum level of ANXA4 might be a promising biomarker for the early detection of hepatocellular carcinoma (39). A recent study indicated that downregulation of ANXA4 by toosendanin could mediate cisplatin sensitization in non-small cell lung cancer (40).

Furthermore, the correlations between immune infiltration and ANXA4 expression levels in various cancer types were calculated. TIMER and xCell suggested that ANXA4 were markedly positively correlated with tumor infiltrating lymphocytes in most cancer types, affecting tumor development via tumor microenvironment (41-43). CTLA-4 and PD-1 inhibitors are immune checkpoint inhibitors showing acceptable results in lung cancer and melanoma. We collected 40 common immune checkpoints and examined the associations between ANXA4 expression levels and immune checkpoints to find its potential as a further therapeutic target in the clinic. Moreover, a 21-genes PPI network centered on ANXA4 was constructed. The enrichment analysis demonstrated that these genes were enriched in prostaglandin synthesis and regulation, negative regulation of phospholipase A2 activity, response to calcium ion, and response to radiation. Prostaglandin is related to inflammation, which plays a crucial role in both $\mathrm{AF}$ and cancer (44-46). GSEA results indicated that the high ANXA4 expression group was mainly enriched in peroxisome, bile acid, and p53 pathway, which were all associated with different cancer types.

Although the integrative analysis on ANXA4 was performed using different databases and algorithms, there are some limitations in the present study. First, ANXA4 has never been reported in AF. The further experiment should be performed to explore and verify its potential mechanism in AF. Second, ANXA4 was identified through proteomics analysis and verified using qRT-PCR. However, the roles of ANXA4 in cancers were analyzed solely based on our bioinformatics analysis, whereas in vitro or in vivo experiments should be conducted to elucidate the molecular mechanisms of ANXA4 in different cancer types. Third, further prospective studies on cancer patients with AF should be carried out to evaluate the efficacy of ANXA4 in reducing the incidence of AF in cancer patients.

\section{CONCLUSION}

In short, the present study identified ANXA4 as a hub immunerelated gene, which has never been reported in AF. The pan-cancer analysis suggested that ANXA4 was significantly associated with prognosis and immune infiltration in various cancer types, which has the potential as a novel biomarker in cancer patients. Considering its role in $\mathrm{AF}$ and cancer, targeted therapy for ANXA4 might reduce the incidence of AF in cancer patients.

\section{DATA AVAILABILITY STATEMENT}

The datasets presented in this study can be found in online repositories. The names of the repository/repositories and accession number(s) can be found in the article/Supplementary Material.

\section{ETHICS STATEMENT}

The studies involving human participants were reviewed and approved by Medical Ethics Committee of East Hospital, Tongji 
University. The patients/participants provided their written informed consent to participate in this study.

\section{AUTHOR CONTRIBUTIONS}

CG, CW, and YZ designed the experiments and analyzed data. TY, SZ, and YS analyzed all the data, wrote the manuscript, and interpreted results. CX and MZ performed data acquisition and part of the data analysis. All authors reviewed the final manuscript and approved it for publication.

\section{REFERENCES}

1. January CT, Wann LS, Calkins H, Chen LY, Cigarroa JE, Cleveland JC, et al. 2019 AHA/ACC/HRS focused update of the 2014 AHA/ACC/HRS guideline for the management of patients with atrial fibrillation: a report of the American college of cardiology/American heart association task force on clinical practice guidelines and the heart rhythm society in collaboration with the society of thoracic surgeons. Circulation. (2019) 140:104-32. doi: 10.1161/CIR.0000000000000719

2. Fang MC, Go AS, Chang Y, Borowsky L, Pomernacki NK, Singer DE. Comparison of risk stratification schemes to predict thromboembolism in people with nonvalvular atrial fibrillation. J Am Coll Cardiol. (2008) 51:8105. doi: 10.1016/j.jacc.2007.09.065

3. Hindricks G, Potpara T, Dagres N, Arbelo E, Bax JJ, Blomström-Lundqvist C, et al. 2020 ESC Guidelines for the diagnosis and management of atrial fibrillation developed in collaboration with the European Association for Cardio-Thoracic Surgery (EACTS). Eur Heart J. (2021) 42:373498. doi: 10.1093/eurheartj/ehaa612

4. Bray F, Ferlay J, Soerjomataram I, Siegel RL, Torre LA, Jemal A. Global cancer statistics 2018: GLOBOCAN estimates of incidence and mortality worldwide for 36 cancers in 185 countries. CA Cancer J Clin. (2018) 68:394424. doi: 10.3322/caac. 21492

5. PS Yang, M Shim, SH Kang, SH Kim, WJ Kim, JY Moon, et al. Incidence of atrial fibrillation in cancer patients according to cancer type. The European Society of Cardiology Congress (2020). Available online at: https://esc365. escardio.org/Congress/ESC-CONGRESS-2020-The-Digital-Experience/ Cardiovascular-Disease-in-Special-Populations-ePosters/219418-incidenceof-atrial-fibrillation-in-cancer-patients-according-to-cancer-type\#abstract. (accessed April 21, 2021).

6. Hernandez MA, Moro C. Atrial fibrillation and C-reactive protein: searching for local inflammation. J Am Coll Cardiol. (2007) 49:164950. doi: 10.1016/j.jacc.2007.02.009

7. Liu L, Zheng Q, Lee J, Ma Z, Zhu Q, Wang Z. PD-1/PD-L1 expression on $\mathrm{CD}(4+) \mathrm{T}$ cells and myeloid DCs correlates with the immune pathogenesis of atrial fibrillation. J Cell Mol Med. (2015) 19:1223-33. doi: 10.1111/jcmm.12467

8. Frustaci A, Chimenti C, Bellocci F, Morgante E, Russo MA, Maseri A. Histological substrate of atrial biopsies in patients with lone atrial fibrillation. Circulation. (1997) 96:1180-4. doi: 10.1161/01.CIR.96.4.1180

9. Yan T, Zhu S, Zhu M, Wang C, Guo C. Integrative identification of hub genes associated with immune cells in atrial fibrillation using weighted gene correlation network analysis. Front Cardiovasc Med. (2020) 7:631775. doi: $10.3389 /$ fcvm.2020.631775

10. Ryschich E, Notzel T, Hinz U, Autschbach F, Ferguson J, Simon I, et al. Control of T-cell-mediated immune response by HLA class I in human pancreatic carcinoma. Clin Cancer Res. (2005) 11:498-504. Available online at: https:// clincancerres.aacrjournals.org/content/11/2/498

11. Barretina J, Caponigro G, Stransky N, Venkatesan K, Margolin AA, Kim S, et al. The Cancer Cell Line Encyclopedia enables predictive modelling of anticancer drug sensitivity. Nature. (2012) 483:603-7. doi: 10.1038/nature11003

12. The Genotype-Tissue Expression (GTEx) project. Nat Genet. (2013) 45:58085. doi: $10.1038 /$ ng.2653

\section{FUNDING}

This study was supported by the General Program of the National Natural Science Foundation of China (No. 81770408).

\section{SUPPLEMENTARY MATERIAL}

The Supplementary Material for this article can be found online at: https://www.frontiersin.org/articles/10.3389/fcvm. 2021.713983/full\#supplementary-material

13. Breuer K, Foroushani AK, Laird MR, Chen C, Sribnaia A, Lo R, et al. InnateDB: systems biology of innate immunity and beyond-recent updates and continuing curation. Nucleic Acids Res. (2013) 41:D122833. doi: $10.1093 /$ nar/gks1147

14. Liu B, Li X, Zhao C, Wang Y, Lv M, Shi X, et al. Proteomic analysis of atrial appendages revealed the pathophysiological changes of atrial fibrillation. Front Physiol. (2020) 11:573433. doi: 10.3389/fphys.2020.573433

15. Zhang B, Horvath S, A. general framework for weighted gene co-expression network analysis. Stat Appl Genet Mol Biol. (2005) 4:e17. doi: 10.2202/1544-6115.1128

16. Dennis GJ, Sherman BT, Hosack DA, Yang J, Gao W, Lane HC, et al. Database for annotation, visualization, and integrated discovery. Genome Biol. (2003) 4:P3. doi: 10.1186/gb-2003-4-9-r60

17. Zhou Y, Zhou B, Pache L, Chang M, Khodabakhshi AH, Tanaseichuk O, et al. Metascape provides a biologist-oriented resource for the analysis of systemslevel datasets. Nat Commun. (2019) 10:1523. doi: 10.1038/s41467-019-09234-6

18. Warde-Farley D, Donaldson SL, Comes O, Zuberi K, Badrawi $\mathrm{R}$, Chao $\mathrm{P}$, et al. The GeneMANIA prediction server: biological network integration for gene prioritization and predicting gene function. Nucleic Acids Res. (2010) 38:W214-20. doi: 10.1093/nar/gkq 537

19. Subramanian A, Tamayo P, Mootha VK, Mukherjee S, Ebert BL, Gillette MA, et al. Gene set enrichment analysis: a knowledge-based approach for interpreting genome-wide expression profiles. Proc Natl Acad Sci U S A. (2005) 102:15545-50. doi: 10.1073/pnas.0506580102

20. Li T, Fan J, Wang B, Traugh N, Chen Q, Liu JS Li B, et al. A web server for comprehensive analysis of tumor-infiltrating immune cells. Cancer Res. (2017) 77:e108-10. doi: 10.1158/0008-5472.CAN-17-0307

21. Aran D, Hu Z, Butte AJ. xCell: digitally portraying the tissue cellular heterogeneity landscape. Genome Biol. (2017) 18:220. doi: 10.1186/s13059-017-1349-1

22. Yoshihara K, Shahmoradgoli M, Martinez E, Vegesna R, Kim H, Torres-Garcia W, et al. Inferring tumour purity and stromal and immune cell admixture from expression data. Nat Commun. (2013) 4:2612. doi: $10.1038 /$ ncomms 3612

23. Vergara P, Della BP. Management of atrial fibrillation. F1000Prime Rep. (2014) 6:22. doi: 10.12703/P6-22

24. Huxley RR, Lopez FL, Folsom AR, Agarwal SK, Loehr LR, Soliman EZ, et al. Absolute and attributable risks of atrial fibrillation in relation to optimal and borderline risk factors: the Atherosclerosis Risk in Communities (ARIC) study. Circulation. (2011) 123:1501-8. doi: 10.1161/CIRCULATIONAHA.110.00 9035

25. Kato T, Yamashita T, Sekiguchi A, Sagara K, Takamura M, Takata S, et al. What are arrhythmogenic substrates in diabetic rat atria? J Cardiovasc Electrophysiol. (2006) 17:890-4. doi: 10.1111/j.1540-8167.2006.00528.x

26. Mason FE, Pronto J, Alhussini K, Maack C, Voigt N. Cellular and mitochondrial mechanisms of atrial fibrillation. Basic Res Cardiol. (2020) 115:72. doi: 10.1007/s00395-020-00827-7

27. Bertero E, Maack C. Calcium signaling and reactive oxygen species in mitochondria. Circ Res. (2018) 122:146078. doi: 10.1161/CIRCRESAHA.118.310082 
28. Sharma S, Sharma G, Hote M, Devagourou V, Kesari V, Arava S, et al. Light and electron microscopic features of surgically excised left atrial appendage in rheumatic heart disease patients with atrial fibrillation and sinus rhythm. Cardiovasc Pathol. (2014) 23:319-26. doi: 10.1016/j.carpath.2014.07.008

29. Emelyanova L, Ashary Z, Cosic M, Negmadjanov U, Ross G, Rizvi F, et al. Selective downregulation of mitochondrial electron transport chain activity and increased oxidative stress in human atrial fibrillation. Am J Physiol Heart Circ Physiol. (2016) 311:H54-63. doi: 10.1152/ajpheart.00699.2015

30. Tu T, Zhou S, Liu Z, Li X, Liu Q. Quantitative proteomics of changes in energy metabolism-related proteins in atrial tissue from valvular disease patients with permanent atrial fibrillation. Circ J. (2014) 78:9931001. doi: 10.1253/circj.CJ-13-1365

31. Murphy MP. How mitochondria produce reactive oxygen species. Biochem J. (2009) 417:1-13. doi: 10.1042/BJ20081386

32. Friedrichs $\mathrm{K}$, Baldus $\mathrm{S}$, Klinke A. Fibrosis in Atrial Fibrillation - Role of Reactive Species and MPO. Front Physiol. (2012) 3:214. doi: 10.3389/fphys.2012.00214

33. Martinez-Reyes I, Cardona LR, Kong H, Vasan K, McElroy GS, Werner M, et al. Mitochondrial ubiquinol oxidation is necessary for tumour growth. Nature. (2020) 585:288-92. doi: 10.1038/s41586-020-2475-6

34. Weinberg SE, Singer BD, Steinert EM, Martinez CA, Mehta MM, MartinezReyes I, et al. Mitochondrial complex III is essential for suppressive function of regulatory T cells. Nature. (2019) 565:495-9. doi: 10.1038/s41586-018-0846-z

35. Roth KG, Mambetsariev I, Kulkarni P, Salgia R. The Mitochondrion as an Emerging Therapeutic Target in Cancer. Trends Mol Med. (2020) 26:11934. doi: 10.1016/j.molmed.2019.06.009

36. Heinick A, Pluteanu F, Hermes C, Klemme A, Domnik M, Husser X, et al. Annexin A4 N-terminal peptide inhibits adenylyl cyclase 5 and limits betaadrenoceptor-mediated prolongation of cardiac action potential. FASEB J. (2020) 34:10489-504. doi: 10.1096/fj.201902094RR

37. Liu J, Wang $H$, Zheng $M$, Deng L, Zhang $X$, Lin $B$. p53 and ANXA4/NFkappaB p50 complexes regulate cell proliferation, apoptosis and tumor progression in ovarian clear cell carcinoma. Int J Mol Med. (2020) 46:2102-14. doi: 10.3892/ijmm.2020.4757

38. Liu J, Zheng M, Qi Y, Wang H, Liu M, Liu Q, et al. Lewis(y) antigen-mediated positive feedback loop induces and promotes chemotherapeutic resistance in ovarian cancer. Int J Oncol. (2018) 53:1774-86. doi: 10.3892/ijo.2018.4496

39. Saad ZM, Fouad Y, Ali LH, Hassanin TM. Clinical significance of annexin A4 as a biomarker in the early diagnosis of hepatocellular carcinoma. Asian Pac J Cancer Prev. (2020) 21:2661-5. doi: 10.31557/APJCP.2020.21.9.2661

40. Zheng MD, Wang ND Li XL, Yan J, Tang JH, Zhao XH, Zhang Z. Toosendanin mediates cisplatin sensitization through targeting Annexin
A4/ATP7A in non-small cell lung cancer cells. J Nat Med. (2018) 72:72433. doi: 10.1007/s11418-018-1211-0

41. Solinas C, Pusole G, Demurtas L, Puzzoni M, Mascia R, Morgan G, et al. Tumor infiltrating lymphocytes in gastrointestinal tumors: controversies and future clinical implications. Crit Rev Oncol Hematol. (2017) 110:10616. doi: 10.1016/j.critrevonc.2016.11.016

42. Yu J, Green MD Li S, Sun Y, Journey SN, Choi JE, et al. Liver metastasis restrains immunotherapy efficacy via macrophage-mediated $\mathrm{T}$ cell elimination. Nat Med. (2021) 27:152-64. doi: 10.1038/s41591-020-1131-x

43. Jerby-Arnon L, Neftel C, Shore ME, Weisman HR, Mathewson ND, McBride MJ, et al. Opposing immune and genetic mechanisms shape oncogenic programs in synovial sarcoma. Nat Med. (2021) 27:289300. doi: 10.1038/s41591-020-01212-6

44. Liang X, Wang Q, Shi J, Lokteva L, Breyer RM, Montine TJ, et al. The prostaglandin E2 EP2 receptor accelerates disease progression and inflammation in a model of amyotrophic lateral sclerosis. Ann Neurol. (2008) 64:304-14. doi: 10.1002/ana.21437

45. Karam BS, Chavez-Moreno A, Koh W, Akar JG, Akar FG. Oxidative stress and inflammation as central mediators of atrial fibrillation in obesity and diabetes. Cardiovasc Diabetol. (2017) 16:120. doi: 10.1186/s12933-017-0604-9

46. Trinchieri G. Cancer and inflammation: an old intuition with rapidly evolving new concepts. Annu Rev Immunol. (2012) 30:677-706. doi: 10.1146/annurev-immunol-020711-075008

Conflict of Interest: The authors declare that the research was conducted in the absence of any commercial or financial relationships that could be construed as a potential conflict of interest.

Publisher's Note: All claims expressed in this article are solely those of the authors and do not necessarily represent those of their affiliated organizations, or those of the publisher, the editors and the reviewers. Any product that may be evaluated in this article, or claim that may be made by its manufacturer, is not guaranteed or endorsed by the publisher.

Copyright (c) 2021 Yan, Zhu, Shi, Xie, Zhu, Zhang, Wang and Guo. This is an open-access article distributed under the terms of the Creative Commons Attribution License (CC BY). The use, distribution or reproduction in other forums is permitted, provided the original author(s) and the copyright owner(s) are credited and that the original publication in this journal is cited, in accordance with accepted academic practice. No use, distribution or reproduction is permitted which does not comply with these terms. 